Pseudomonasinfektion bei zystischer Fibrose

\title{
Mutatorstämme als Ursache für schlechtere Lungenfunktion?
}

Ferroni A et al. Effect of Mutator P. aeruginosa on Antibiotic Resistance Acquisition and Respiratory Function in Cystic Fibrosis. Pediatr Pulmonol. 2009;44:820-825

\section{Hintergrund}

Patienten mit zystischer Fibrose (CF) haben eine chronische bakterielle Infektion der Lunge mit einer progressiven Verschlechterung der Lungenfunktion. Der dafür verantwortliche Keim ist häufig Pseudomonas aeruginosa.

Resistente Stämme entstehen bei CF-Patienten typischerweise durch $\mathrm{Mu}$ tationen der Erreger innerhalb des Patienten. Bei Patienten ohne eine CF entwickeln sich Resistenzen dagegen meist durch den Erwerb von Resistenzgenen von außen. Einige Stämme (Mutatorstämme) haben eine spontan erhöhte Mutationsrate, meist aufgrund von Defekten im DNS-Reparatursystem. Diese Bakterien haben einen Selektionsvorteil, insbesondere bei der Eroberung neuer Habitate.

\section{Fragestellung}

Ziel der Studie mit 36 CF-Patienten war es, das Resistenzmuster und die Häu- figkeit des Mutatorphänotyps von 136 Pseudomonas-aeruginosa-Stämmen sowie den Zusammenhang zur klinischen Symptomatik über einen 3-Jahres-Zeitraum zu beschreiben.

\section{Ergebnisse}

Der Anteil resistenter Bakterien war innerhalb der Gruppe der Mutatorstämme höher, und zwar durchgängig für alle getesteten Antibiotika.

Die Zeit bis zur Entstehung einer Resistenz gegen Antibiotika war unterschiedlich. Mutatorstämme werden etwa doppelt so schnell resistent wie Nichtmutatorstämme (Abb. 1).

Die Lungenfunktion von Patienten mit Mutatorstämmen war schlechter als die der anderen Patienten (Abb. 2).

\section{Schlussfolgerung}

Mutatorstämme von Pseudomonas aeruginosa erhöhen das Risiko für die Entwicklung antibiotischer Resistenzen.

\section{Kommentar}

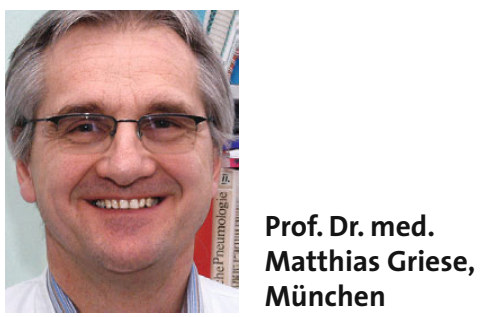

Ob die Resistenzentwicklung von Pseudomonas aeruginosa wirklich eine schlechtere Lungenfunktion bedingt, bleibt in prospektiven Untersuchungen zu klären, da diese Querschnittsbeobachtung nicht berücksichtigt, dass die Therapieintensitäten bei den beiden Patientengruppen mit Mutatorund Nichtmutatorstämmen möglicherweise unterschiedlich waren.

In-vitro-Experimente zeigen, dass manche Antibiotikakombinationen eine höhere therapeutische Effizienz hinsichtlich der Reduktion der Keimlast, der Mortalität und der Entwicklung von Mutatorstämmen haben als andere. Zum Beispiel war Ciprofloxacin + Tobramycin besser als Ceftazidim + Tobramycin oder Ceftazidim + Ciprofloxacin. Daher sind dringend klinische Studien notwendig, um diejenigen Antibiotikakombinationen zu identifizieren, die die Entstehung von Mutatorstämmen, assoziierte Verschlechterungen der Lungenfunktion und eine Resistenzentwicklung am besten vermeiden.

\section{Zeit bis zum Auftreten neuer Resistenzen gegen Pseudomonas aeruginosa \\ Abbildung 1}

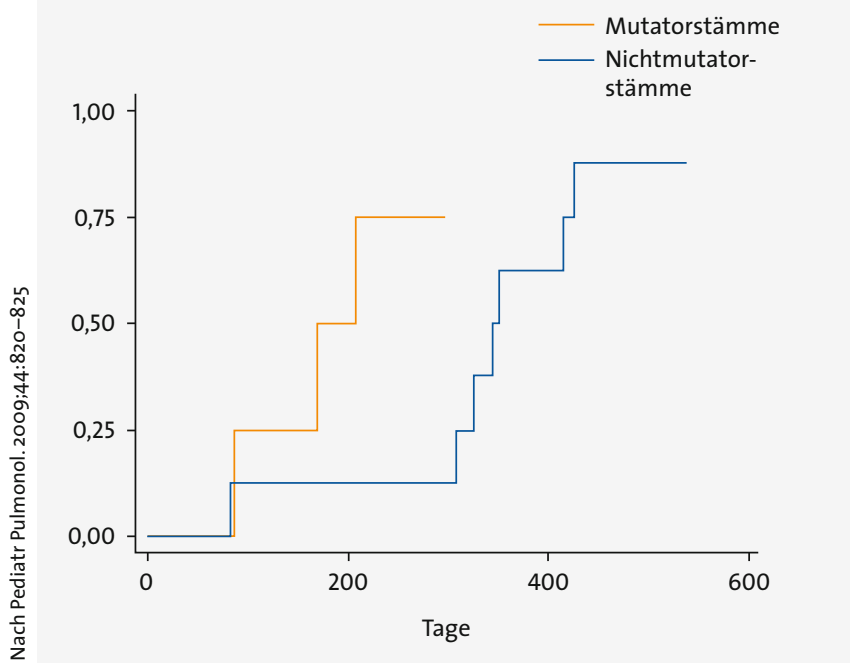

\section{Einsekundenkapazität von CF-Patienten mit oder ohne Mutatorstämme}

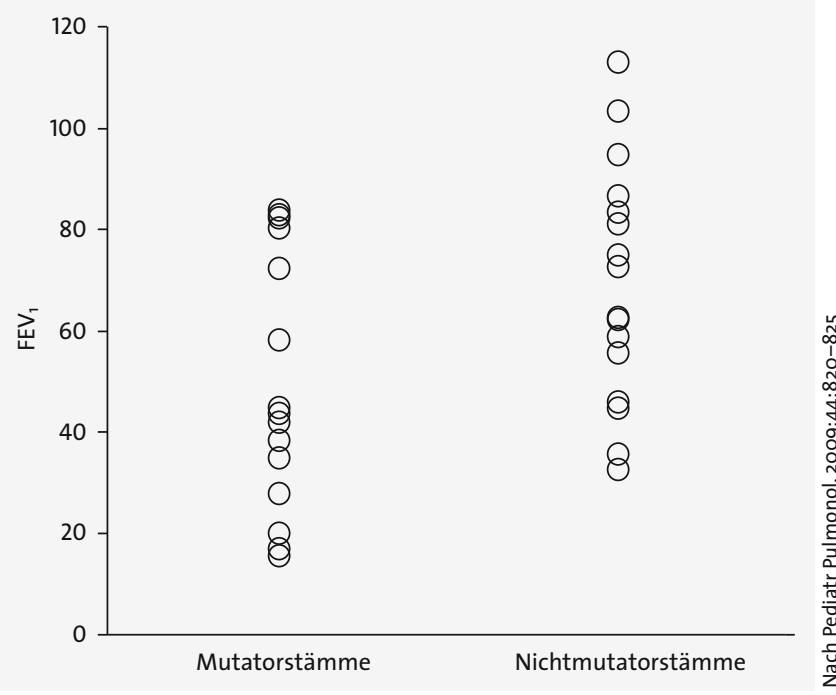

